\title{
Salvage prostatectomy post-definitive radiation therapy: The Vancouver experience
}

\author{
Niall M. Corcoran, MD, PhD; Guilherme Godoy, MD; Rodney C. Studd, MD; Rowan G. Casey, MD;* \\ Antonio Hurtado-Coll, MD; ${ }^{*}$ Scott Tyldesley, MD, FRCPC; ${ }^{+}$S. Larry Goldenberg, MD, FRCSC, FACS; ${ }^{*}$ \\ Martin E. Gleave, MD, FRCSC, FACS
}

"Department of Urological Sciences and Vancouver Prostate Centre, University of British Columbia, Vancouver, BC; 'Department of Radiation Oncology, BC Cancer Agency, Vancouver, BC

See related article on page 93.

Cite as: Can Urol Assoc J 2013;7:87-92. htrp://dx.doi.org/10.5489/cuaj.11056. Epub 2012 Jan 24.

\section{Abstract}

Introduction: Prostate cancer recurrence following primary radiation is common. If the recurrence remains localized to the prostate gland, surgical removal may result in long-term local control or cure. Despite the well-established oncological outcomes, salvage prostatectomy is infrequently performed or reported. We present our experience with salvage prostatectomy at a Canadian centre. Methods: We identified all patients undergoing salvage prostatectomy at the Vancouver General Hospital between 1995 and 2010 from a prospectively recorded and maintained prostate cancer database. Details regarding initial presentation, delivery of radiotherapy, clinical features at the time of recurrence, as well as oncological and functional outcomes, were collected. Information regarding postoperative morbidity was collected prospectively and confirmed by retrospective chart review.

Results: Over a 15-year period, salvage prostatectomy was successfully completed in 21 patients. With a median follow-up period of 68 months (range: $2-122), 9$ (43\%) patients experienced a biochemical recurrence, with most failing within the first 2 years of surgery. There were 3 deaths in the cohort, all from prostate cancer, giving a prostate cancer specific and overall survival of $86 \%$. The main postoperative morbidity was bladder neck contracture, occurring in $40 \%$. One patient each developed a recto-urethral fistula and osteitis pubis. Physician-recorded data regarding continence was available in 13 (62\%). Of these 13 patients, 10 (85\%) men were recorded as dry or using 1 pad per day.

Conclusions: This is the first Canadian centre to report that salvage prostatectomy can be performed with favourable oncological and functional outcomes.

\section{Introduction}

Radiation therapy (RT), either in the form of external beam radiotherapy (EBRT) or brachytherapy, is commonly used to treat localized prostate cancer. Recent analysis of the CaPSURE database indicates that up to $25 \%$ of men presenting in North America choose some form of radiotherapy as their primary treatment. ${ }^{1}$ Similar to other treatment modalities, the risk of disease recurrence is directly related to the extent and grade of disease at presentation, with up to $10 \%$ of low-risk patients and up to $60 \%$ of high-risk patients experiencing disease recurrence in the medium- to long-term. ${ }^{2}$

Several therapeutic options exist to manage locally recurrent prostate cancer post-irradiation, including active surveillance, androgen deprivation therapy (ADT), thermotherapy or cryotherapy, salvage brachytherapy and radical prostatectomy. The last three modalities have been reported to be curative in selected patients, although a number of series report that successful salvage rates are higher with surgery than either cryotherapy or brachytherapy. ${ }^{3-5}$ Although tumour recurrence post-radiotherapy is common, and in more than $70 \%$ is localized to the prostate gland, salvage prostatectomy in infrequently performed. In a survey of clinicians' treatment recommendations for biochemical recurrence postradiotherapy, only $25 \%$ considered salvage prostatectomy the preferred option in patients younger than 65 , which fell to $4 \%$ in patients over this age. ${ }^{6}$ Lack of enthusiasm for prostatectomy as a salvage option probably reflects concerns about the technical difficulties associated with the procedure, as well as higher postoperative morbidity.

In the last decade, there have been a number of contemporary series describing the oncological and functional outcomes of salvage prostatectomy, with very reasonable results reported. ${ }^{5,7,8}$ However, most these reports represent the experience of the three high-volume cancer centres in the United States, and therefore the results may not accurately reflect those of smaller or non-American centres. In particular, the local results of Canadian centres are unknown. 
Corcoran et al.

We report our 15-year experience with salvage radical prostatectomy at the Vancouver General Hospital (Vancouver, BC, Canada).

\section{Methods}

\section{Patient selection}

Patients undergoing salvage prostatectomy following primary RT at the Vancouver General Hospital from 1995 to present were identified from a prospectively recorded and maintained prostate cancer database. Patients undergoing salvage procedures for failure of other treatment modalities (high-intensity focused ultrasound [HIFU], cryotherapy) were excluded. Salvage prostatectomy post-HIFU was performed in only one instance, and no patients were treated post-cyrotherapy. This patient was excluded as we were specifically interested in the outcomes of prostatectomy post-radiation. We also excluded a patient treated postbrachytherapy, in whom a safe plane could not be developed between the prostate gland and the pelvic sidewall at the apex, and the procedure was abandoned.

Recurrence post-radiation was defined according to the American Society for Therapeutic Radiology and Oncology (ASTRO) definition (3 successive rises in serum prostatespecific antigen [PSA] above nadir, with the date of recurrence backdated to the midpoint between the nadir and first measurements). All patients had biopsy-proven recurrence within the prostate gland, with no clinical or radiological (bone scan \pm computed tomography [CT]) evidence of metastatic disease at the time of presentation. Patients considered for prostatectomy were otherwise well, with an estimated life expectancy exceeding 10 years. Surgery consisted of a standard radical retropubic prostatectomy with no attempt made to spare the neurovascular bundles. Unilateral or bilateral lymph node dissection was performed in selected cases. Neoadjuvant ADT was used at the discretion of the treating physician. In patients who received neo-adjuvant hormonal therapy, prostate biopsy confirming the presence of malignancy was obtained prior to commencement.

\section{Data collection}

All clinical and pathological information was recorded prospectively, but analyzed retrospectively. Clinical details regarding the initial presentation, as well as the technical details of radiation delivery, were provided by the British Columbia Cancer Agency (BCCA). Details of the most recent PSA follow-up, as well as vital status, were obtained from chart review, contact with the pathology and primary care providers, as well as interrogation of the BCCA's followup database. For this analysis, biochemical recurrence was defined as a postoperative PSA $>0.2 \mathrm{ng} / \mathrm{mL}$ and rising. Information regarding morbidity was collected from the database and confirmed by chart review. Collection and use of this data had institutional review board approval.

\section{Data analysis}

Data on continuous and categorical variables are presented as medians or means, with their respective ranges. Data on categorical variables are presented as proportions. KaplanMeier curves were generated to visually illustrate cohort biochemical recurrence and overall survival with time. Given the small number of cases, all statistics are purely descriptive.

\section{Results}

From a total of 2024 radical prostatectomy cases performed over the study period, 21 (1\%) cases were performed for cancer recurrence following radiotherapy; their clinical char-

\section{Table 1. Characteristics of study cohort}

$\mathrm{N}=21$

\begin{tabular}{|c|c|c|c|}
\hline \multicolumn{4}{|l|}{ Radiotherapy } \\
\hline Age & Median & 60 (range: 47-70) & \\
\hline PSA $(n=17)$ & Median & 9.2 (range: $0.9-40$ ) & \\
\hline CT $(n=20)$ & $\begin{array}{c}\text { T1 } 3 \\
\text { (15\%) }\end{array}$ & T2 12 (60\%) & $\begin{array}{c}\text { T3 } 5 \\
(25 \%)\end{array}$ \\
\hline \multirow[t]{3}{*}{ Biopsy GS $(n=18)$} & $\leq 6$ & 7 (39\%) & \\
\hline & 7 & $10(56 \%)$ & \\
\hline & 8-10 & $1(5 \%)$ & \\
\hline EBRT dose & Median & 66 Gy (range: 52.5-74) & \\
\hline \multirow[t]{3}{*}{ EBRT fields $(n=19)$} & Rotating & $2(11 \%)$ & \\
\hline & 4 & $16(84 \%)$ & \\
\hline & 5 & $1(5 \%)$ & \\
\hline \multirow[t]{3}{*}{ EBRT phases $(n=19)$} & 1 & $9(47 \%)$ & \\
\hline & 2 & $8(42 \%)$ & \\
\hline & 3 & $2(11 \%)$ & \\
\hline \multirow[t]{2}{*}{ ADT } & No & $14(67 \%)$ & \\
\hline & Yes & 7 (33\%) & \\
\hline PSA nadir $(n=14)$ & Median & 1.1 (range: 0-3.5) & \\
\hline \multicolumn{4}{|l|}{ Prostatectomy } \\
\hline Interval & Median & 4.4 (range: $1.4-11.7$ ) & \\
\hline Preoperative PSA & Mean & 3.5 (range: $0.3-10.1$ ) & \\
\hline CT & $\begin{array}{c}\text { T1 } 8 \\
(38 \%)\end{array}$ & T2 9 (43\%) & $\begin{array}{c}\text { T3 } 4 \\
\text { (19\%) }\end{array}$ \\
\hline \multirow[t]{3}{*}{ Biopsy GS (n=20) } & $\leq 6$ & $8(38 \%)$ & \\
\hline & 7 & $9(43)$ & \\
\hline & $8-10$ & $3(19 \%)$ & \\
\hline \multirow[t]{2}{*}{ Neo-adjuvant ADT } & No & $9(43 \%)$ & \\
\hline & Yes & $12(57 \%)$ & \\
\hline
\end{tabular}

PSA: prostate-specific antigen; CT: computed tomography; GS: Gleason score; EBRT: external beam radiotherapy; $A D T$ : androgen deprivation therapy. 
acteristics are listed (Table 1). According to the D'Amico classification, low-, intermediate- and high-risk disease was present in $5(24 \%), 9(43 \%)$ and $7(33 \%)$ patients, respectively. ${ }^{9}$ All patients received RT in the form of EBRT, and the median interval from treatment to recurrence was over 5 years. The median total dose to the isocentre of the prostate was 66 Gy (range: 52.5-70), usually delivered in 2 Gy fractions. In most patients, a 4-field technique was used, however, a dual partial rotating arc technique was used 2 cases and a 5 -field technique in 1 patient. Radiation was initially delivered to the pelvis in 8 patients, the remaining received RT to the prostate \pm seminal vesicles alone in 1 to 2 phases. The median dose to the pelvis, when given, was 45 Gy (range: 44-46) at 2 Gy per fraction. All treatments were CT planned, with customized conformal blocking in most cases. Twelve (57\%) patients received neo-adjuvant ADT for a median of 8 months (range: 4-8) preoperatively.

The pathological characteristics of the cohort were noted (Table 2). Fifteen (71\%) patients had organ-confined disease, and the positive surgical margin rate was 19\%. Eight patients underwent a lymph node dissection, which confirmed the presence of metastatic disease in 1 patient. This patient experienced a rapid biochemical recurrence and died from his disease within 4 years of the procedure.

With a median follow-up period of 68 months (range: 2-122), 9 (43\%) patients experienced a biochemical recurrence. Most patients who failed did so in the first 2 years after surgery, consistent with the development of metastatic disease (Fig. 1). After this initial period, the rates of biochemical recurrence were low. With a median follow-up of 81 months (range: 1.2-177), 3 patients died, giving an overall survival rate of $86 \%$ (Fig. 2). All patients who died did so from progressive prostate cancer.

Morbidity experienced by the study group was tallied (Table 3). Data concerning urinary continence were avail-

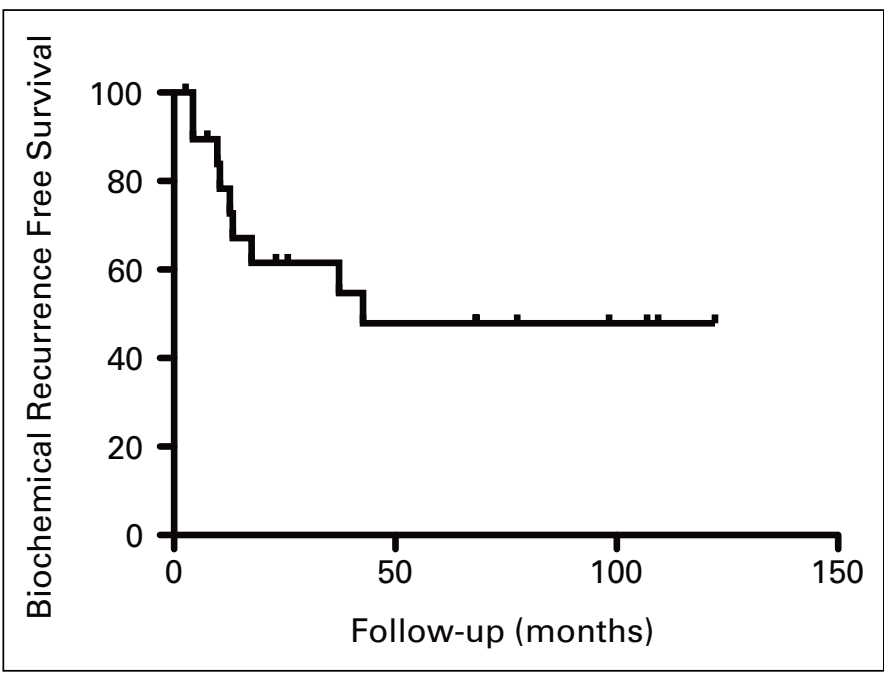

Fig. 1. Biochemical recurrence post salvage prostatectomy.

\begin{tabular}{|c|c|c|}
\hline \multicolumn{3}{|l|}{$\mathrm{N}=21$} \\
\hline $\mathrm{pT}$ & T2 15 (71\%) & T3 6 (29\%) \\
\hline \multirow[t]{4}{*}{ GS } & 6 & $4(19 \%)$ \\
\hline & 7 & $10(48 \%)$ \\
\hline & 8-10 & $4(19 \%)$ \\
\hline & Treatment effect & $3(14 \%)$ \\
\hline \multirow[t]{2}{*}{ EPE } & No & $15(71 \%)$ \\
\hline & Yes & $6(29 \%)$ \\
\hline \multirow[t]{2}{*}{ SVI } & No & $18(86 \%)$ \\
\hline & Yes & $3(14 \%)$ \\
\hline \multirow[t]{2}{*}{ Positive margin } & No & 17 (81\%) \\
\hline & Yes & $4(19 \%)$ \\
\hline \multirow[t]{2}{*}{ PLND } & No & $13(62 \%)$ \\
\hline & Yes & $8(38 \%)$ \\
\hline \multirow[t]{2}{*}{ Positive LN } & No & 7 (88\%) \\
\hline & Yes & $1(12 \%)$ \\
\hline
\end{tabular}

GS: Gleason score; EPE: extraprostatic extension SVI: seminal vesicle involvement; PLND: pelvic lymph node dissection; LN: lymph node.

able for 13 patients, with 10 (77\%) being dry or requiring 1 or fewer pads per day. Data concerning bladder neck contractures were available for 15 patients, with $6(40 \%)$ developing significant stenosis requiring intervention (complications were classified according to the Clavien system ${ }^{10}$ ). In addition, there was 1 case of occult rectal injury at the time of surgery that presented in the early postoperative period with a recto-urethral fistula, as well 1 case of bacterial osteitis pubis that required treatment with a prolonged course of antibiotics.

\section{Discussion}

The management of patients with recurrent prostate cancer after definitive primary radiotherapy is extremely challeng-

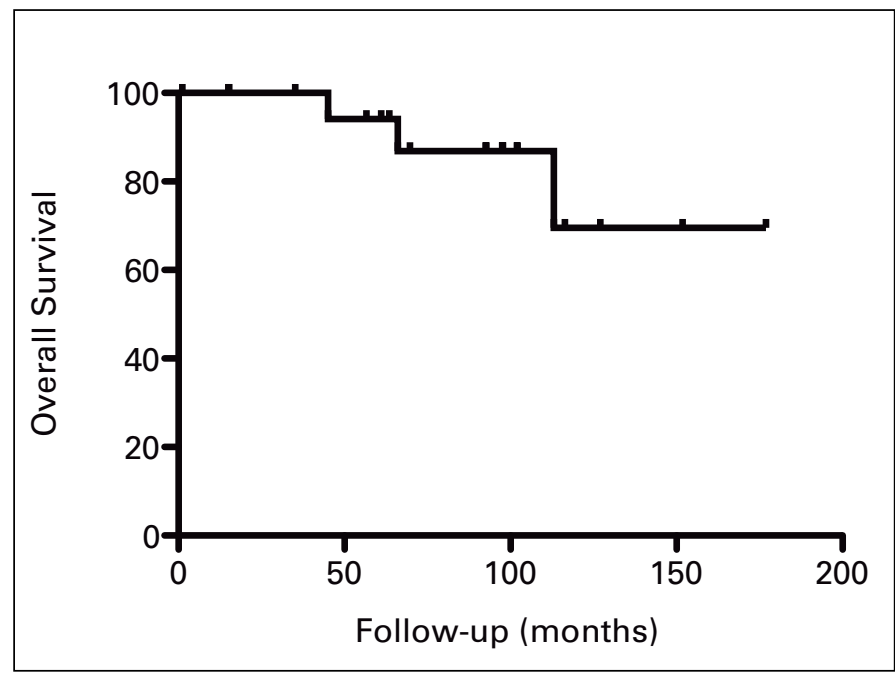

Fig. 2. Overall survival post-prostatectomy. 
Corcoran et al.

\begin{tabular}{|c|c|c|c|c|}
\hline $\mathbf{n}$ & & 13 & & \\
\hline \multirow[t]{2}{*}{ Incontinence } & No & $7(54 \%)$ & & \\
\hline & Yes & $6(46 \%)$ & & \\
\hline 1 pad & 3 & & & \\
\hline $2-3$ pads & 2 & & & \\
\hline$>3$ pads & 1 & & & \\
\hline \multicolumn{5}{|l|}{ Severity } \\
\hline $\mathrm{n}$ & & 15 & Illa & $\mathrm{Illb}$ \\
\hline \multirow[t]{2}{*}{ Bladder neck contracture } & No & $9(60 \%)$ & & \\
\hline & Yes & $6(40 \%)$ & $2(13 \%)$ & $4(27 \%)$ \\
\hline Recto-urethral fistula & & 1 & 1 & \\
\hline Osteitis pubis & & 1 & 1 & \\
\hline
\end{tabular}

ing. Although reports of salvage prostatectomy outside of large American institutions are infrequent, with a median follow-up of over 5 years, we have shown that locally we can achieve oncological outcomes that compare favourably with acceptable morbidity. Even though salvage prostatectomies are rarely performed at our institution, the surgeons involved perform a high volume of standard prostatectomies each year (150-200 cases per year), with a particular interest in the management of high-risk and/or locally advanced cancers, which probably contributes significantly to observed outcomes.

The key to successful salvage treatment is early identification of treatment failure when the disease is more likely to be confined to the prostate gland. Given that in post-RT, failure is primarily based on follow-up serum PSA levels, this can be extremely difficult to do. In primary surgery, where serum PSA should become undetectable with complete removal of the gland, the appearance of a detectable PSA significantly predicts the development of metastases and death. In contrast with primary radiotherapy, the prostate gland remains in situ. There, PSA continues to be produced by benign elements and so remains in the detectable range in most patients. ${ }^{11}$ Not only are these levels subject to normal fluctuations, they are susceptible to the same disorders that affect the normal aging prostate and cause PSA elevations in the absence of recurrence. Interpretation of follow-up PSA is further complicated by the well-recognized "PSAbounce" phenomenon that occurs in up to $20 \%$ of patients. ${ }^{12}$ This intrinsic variability in PSA has complicated an early definition of biochemical failure post-radiotherapy when the institution of salvage therapy may be at its most effective. For most of the study period, the older ASTRO definition of 3 successive rises in serum PSA above nadir defined biochemical recurrence, with the date of recurrence backdated to the midpoint between the nadir and first measurements. In most cases, this definition has been abandoned due to its lack of sensitivity and specificity, and replaced by the Phoenix definition of "nadir +2 ," which has been shown to predict the development of metastases and death. ${ }^{12}$ One criticism of the Phoenix definition is that given it is a strong predictor of cancer-specific mortality, its use clinically to define the need for salvage therapy would mean that by the time treatment failure is declared, the patient is already at significant risk of occult spread and ultimate failure of salvage treatment. In this regard, of the 12 patients in our cohort with paired preoperative and nadir PSA data, $10(83 \%)$ met the Phoenix definition, with a median PSA rise of $3.1 \mathrm{ng} / \mathrm{L}$ (range: $2.4-5$ ). Certainly, most patients who experienced biochemical failure post-salvage prostatectomy did so within the first 18 to 24 months of surgery, a pattern consistent with the presence of occult metastatic disease at the time of operation.

Even if a PSA-based definition of failure was abandoned, it is difficult to envisage a reliable and acceptable alternative. The very nature by which radiation treatment exerts its anti-cancer effect on cancer cells, through the progressive accumulation of DNA damage until eventual cellular collapse, means that there will invariably be a window of time whereby the detection of viable cancer post-treatment does not necessarily predict treatment failure. This is highlighted by the observation up to $30 \%$ of positive biopsies at 12 months post-treatment will convert to negative status by 24 to 30 months. ${ }^{13}$ One alternative would be the routine biopsy of all patients at 2 years post-radiation, as the presence of viable tumour in the absence of treatment effect has been shown to be a strong predictor of subsequent biochemical recurrence in a number of studies. ${ }^{14,15}$ However, the histological changes of radiation treatment effect can be difficult to differentiate from residual viable tumour, and transrectal biopsy carries its own risk of significant morbidity, so routine re-biopsy in all patients is hard to justify. ${ }^{16,17}$ An alternative is to use some form of PSA metric, such as absolute PSA level at nadir, time to PSA recurrence or PSA doubling time, to riskstratify those most likely to fail; the utility of this approach has yet to be determined. Perhaps a more elegant solution will emerge from the ongoing investigations of functional imaging to allow a non-invasive method to identify and localize residual viable cancer. ${ }^{18}$

Even when the PSA begins to rise 12 to 18 months after radiotherapy and recurrent disease is suspected, it is extremely difficult to differentiate local from systemic recurrence, and most patients who recur following salvage prostatectomy do so from occult metastatic disease rather than local treatment failure.

Given that serum PSA levels are usually $<10 \mathrm{ng} / \mathrm{mL}$ at the time of consideration for salvage treatment, the sensitivity of bone scan and CT to detect metastatic disease is low. ${ }^{19}$ Perhaps the best predictor of systemic failure is the pretreatment risk stratification, with $\mathrm{D}^{\prime}$ Amico high-risk patients less likely to experience local recurrence only..$^{13}$ Although our cohort was too small to perform a meaningful analysis of predictors of recurrence post-salvage prostatectomy, larger 
studies have identified preoperative PSA levels and biopsy Gleason score as significant predictors of salvage failure. ${ }^{8}$

It is interesting to note that despite the frequency with which radiation treatment is used and treatment failures occur, very few salvage prostatectomies are actually performed. Extrapolating from the published data of the nearby BCCA, during the course of this study, about 4500 men would have been treated primarily with some form of radiotherapy, and up to 1500 recurrences documented. Of this number, only $22(0.01 \%)$ were selected for salvage prostatectomy, despite well-established significant long-term oncological outcomes in both our own and other published series..$^{3-5}$ As the number of patients referred for consideration of salvage prostatectomy is unknown, it is difficult to determine if this represents failure of referral or unsuitability for salvage. Certainly, many of the men undergoing primary radiotherapy may have been unsuitable for radical surgery from the start, either due to patient age, comorbidity or a desire to avoid an operation, and so salvage surgery may not have been considered. Similarly, a significant number may have been deemed at high risk of systemic failure, either due to their pre-treatment tumour characteristics, rapid time to biochemical recurrence and/or rapid rise in PSA, or the absence of viable tumour on their prostate biopsy. However, the cohort of men who may have benefited from salvage prostatectomy is undoubtedly much larger than that which actually underwent the procedure, which points to a certain perceived bias against the procedure. Much of this has to do with the associated procedural morbidity, which as in our series is significantly higher than that reported for standard prostatectomy. That said, in our hands, over half of men were completely dry with a good urinary flow postoperatively, with most men with ongoing urinary dysfunction having mild symptoms only.

There are a number of significant limitations to this study. Firstly the numbers in the study are low, reflecting the rarity with which the procedure is performed, and this precludes the drawing of definitive conclusions. Although all the data were collected prospectively, they were analyzed retrospectively, with all the limitations this engenders. In particular, functional outcomes are those reported by the patient to the treating physician at the time of clinical review. Although in recent years we have moved to formal patient-reported outcome questionnaires for follow-up, this was not the case earlier in the series, and so functional status as recorded by the physician at the time of clinical review was used. We accept that this may overestimate the true outcomes achieved, particularly with respect to continence. Even for the continence data, we were only able to report on data from $13 / 21$ (62\%) of this study cohort. Given the long period of time over which the study is based, the fact that many of the medical records are now expunged, and that a number of patients have died, moved interstate or overseas, we are reliant on the prospectively recorded data in our database to give us insights into functional recovery; although we recognize that this is limited. In addition, there were too few data recorded in the database and on chart review to report meaningfully on erectile function, although for the small number of patients for which data is available the outcomes are usually poor. We presume that because the neurovascular bundles were widely excised in every case, all patients had prior radical RT and most were treated with ADT, erectile dysfunction was assumed and not a focus of the clinical review. We also recognize that the use of neoadjuvant hormonal therapy (NHT) prior to prostatectomy is not standard therapy. However, at the time that most patients were treated, our unit was specifically interested in the hypothesis that NHT could reduce positive surgical margin rate and the risk of biochemical recurrence postprostatectomy, particularly in high-risk patients. In 2001, we published the initial report of a randomized phase III trial comparing 3 to 8 months of NHT demonstrating that 8 months of NHT resulted in a significantly lower positive surgical margin rate and, therefore, NHT was frequently used "off-label" in high-risk patients. ${ }^{20}$ Prostate cancer specific mortality is defined by the listed causes of death on the death certificate. We recognize that this may under or overestimate prostate cancer specific mortality due to assignment bias. While we can confirm that those who died progressed on ADT, we are unable to do so for chemotherapy. Two of the 3 men who died did so very early in the series, when chemotherapy was palliative in nature, and many patients died from the disease without receiving it. However, despite these limitations we feel this study offers data supporting the concept that radical prostatectomy can be safely performed at a Canadian centre with acceptable oncological and functional outcomes.

\section{Conclusion}

Salvage prostatectomy can be performed outside large quaternary referral centres with acceptable oncological and functional outcomes. Given its well-established rates of cancer control, salvage prostatectomy should be considered the preferred option in managing local recurrence following radiation therapy in carefully selected men.

Acknowledgements: Dr. Corcoran is the recipient of the Hugh Johnston Travelling Fellowship from the Royal Australasian College of Surgeons, as well as a Urological Society of Australia and New Zealand travel grant.

Competing interests: None declared.

This paper has been peer-reviewed. 


\section{References}

1. Dindo D, Demartines N, Clavien PA. Classification of surgical complications: a new proposal with evaluation in a cohort of 6336 patients and results of a survey. Ann Surg 2004;240:205-13. http://dx.doi. org/10.1097/01.sla.0000133083.54934.ae

2. Cooperberg MR, Broering JM, Carroll PR. Time trends and local variation in primary treatment of localized prostate cancer. J Clin Oncol 2010;28:1117-23. http://dx.doi.org/10.1200/JC0.2009.26.0133

3. Boukaram C, Hannoun-Levi JM. Management of prostate cancer recurrence after definitive radiation therapy. Cancer Treat Rev 2010;36:91-100. http://dx.doi.org/10.1016/i.ctrv.2009.06.006

4. Bianco FJ Jr, Scardino PT, Stephenson AJ, et al. Long-term oncologic results of salvage radical prostatectomy for locally recurrent prostate cancer after radiotherapy. Int I Radiat Oncol Biol Phys 2005;62:448-53. http://dx.doi.org/10.1016/i.i.jobp.2004.09.049

5. Pisters LL. Salvage radical prostatectomy: refinement of an effective procedure. Semin Radiat Oncol 2003;13:166-74. http://dx.doi.org/10.1016/S1053-4296(03)70010-1

6. Pisters LL, Leibovici $D$, Blute $M$, et al. Locally recurrent prostate cancer after initial radiation therapy: a comparison of salvage radical prostatectomy versus cryotherapy. J Urol 2009; 182:517-25; discussion 525-7. http://dx.doi.org/10.1016/i.juro.2009.04.006

7. Sylvester J, Grimm P, Blasco J, et al. The role of androgen ablation in patients with biochemical or local failure atter definitive radiation therapy: a survey of practice patterns of urologists and radiation oncologists in the United States. Urology 2001;58:65-70. http://dx.doi.org/10.1016/S0090-4295(01)01244-4

8. Paparel $P$, Cronin AM, Savage $C$, et al. Oncologic outcome and patterns of recurrence after salvage radical prostatectomy. Eur Urol 2009;55:404-10. http://dx.doi.org/10.1016/j.eururo.2008.07.007

9. Ward JF, Sebo TJ, Blute ML, et al. Salvage surgery for radiorecurrent prostate cancer: contemporary outcomes. J Urol 2005;173:1156-60. http://dx.doi.org/10.1097/01.ju.0000155534.54711.60

10. D'Amico AV, Whittington R, Malkowicz SB, et al. Pretreatment nomogram for prostate-specific antigen recurrence after radical prostatectomy or external-beam radiation therapy for clinically localized prostate cancer. J Clin Oncol 1999;17:168-72.

11. Pound CR, Partin AW, Eisenberger MA, et al. Natural history of progression after PSA elevation following radical prostatectomy. JAMA 1999;281:1591-7. http://dx.doi.org/10.1001/jama.281.17.1591

12. Roach $M$ 3rd, Hanks $G$, Thames $H$, Jr., et al. Defining biochemical failure following radiotherapy with or without hormonal therapy in men with clinically localized prostate cancer: recommendations of the RTOGASTRO Phoenix Consensus Conference. Int J Radiat Oncol Biol Phys 2006;65:965-74. http://dx.doi. org/10.1016/i.i.jrobp.2006.04.029
13. Nguyen PL, D'Amico AV, Lee AK, et al. Patient selection, cancer control, and complications after salvage local therapy for postradiation prostate- specific antigen failure: a systematic review of the literature. Cancer 2007;110:1417-28. http://dx.doi.org/10.1002/cncr.22941

14. Crook JM, Malone S, Perry G, et al. Twenty-four-month postradiation prostate biopsies are strongly predictive of 7-year disease-free survival: results from a Canadian randomized trial. Cancer 2009;115:673-9. http://dx.doi.org/10.1002/cncr.24020

15. Zapatero A, Minguez R, Nieto $S$, et al. Post-treatment prostate biopsies in the era of three-dimensional conformal radiotherapy: what can they teach us? Eur Urol 2009;55:902-9. http://dx.doi.org/10.1016/i. eururo.2008.04.076

16. Bostwick DG, Egbert BM, Fajardo LF. Radiation injury of the normal and neoplastic prostate. Am I Surg Pathol 1982;6:541-51. http://dx.doi.org/10.1097/00000478-198209000-00006

17. Nam RK, Saskin R, Lee $Y$, et al. Increasing hospital admission rates for urological complications after transrectal ultrasound guided prostate biopsy. J Urol 2010;183:963-8. http://dx.doi.org/10.1016/i. juro.2009.11.043

18. Sciarra A, Barentsz J, Biartell A, et al. Advances in Magnetic Resonance Imaging: How They Are Changing the Management of Prostate Cancer. Eur Urol 2011;59:962-77. Epub 2011 Feb 23. http://dx.doi. org/10.1016/i.eururo.2011.02.034

19. Gleave ME, Coupland D, Drachenberg D, et al. Ability of serum prostate-specific antigen levels to predict normal bone scans in patients with newly diagnosed prostate cancer. Urology 1996;47:708-12. http:// dx.doi.org/10.1016/S0090-4295(96)80016-1

20. Gleave ME, Goldenberg SL, Chin JL, et al. Randomized comparative study of 3 versus 8-month neoadjuvant hormonal therapy before radical prostatectomy: biochemical and pathological effects. I Urol 2001;166:5006; discussion 506-7. http://dx.doi.org/10.1016/S0022-5347(05)65971-X

Correspondence: Dr. Niall Corcoran, Department of Urological Sciences, 6th Floor Leslie and Gordon Diamond Health Care Centre, 2775 Laurel St., Vancouver, BC V5Z 1M9; fax: 604-875-5604; niallmcorcoran@gmail.com 